\title{
Giro pragmático y teoría política. Un análisis desde la democracia deliberativa ${ }^{1}$
}

Santiago Prono*

Resumen: La relación entre pragmatismo y teoría política viene siendo explorada de diversas maneras en numerosos trabajos que analizan el tema. La idea es explicitar qué recepción lleva a cabo la filosofía política de los presupuestos del pragmatismo. En este contexto, en el presente trabajo se analiza el tema desde la democracia deliberativa de J. Habermas, señalando de qué modo esta teoría política recepta tales presupuestos teniendo en cuenta el marco teórico del discurso argumentativo, que constituye la base filosófica sobre la cual fundamenta su estructura teórica. Como resultado de este análisis es posible señalar (parte de) las contribuciones de aquella teoría política para el mejoramiento de la calidad democrática del estado de derecho.

Palabras clave: Giro pragmático, Discurso, Democracia deliberativa, Reconstrucción racional, Crítica.

Abstract: The relationship between pragmatism and political theory has been explored in different ways by numerous studies that consider the topic in order to explain what kind of reception makes the political philosophy of the presuppositions of pragmatism. In this context, this paper analyzes the issue from the point of view of the deliberative democracy of J. Habermas, indicating how this political theory incorporates such presuppositions bearing in mind the theoretical framework of argumentative discourse, which it is the philosophical background on which bases its theoretical structure. As a result of this analysis it is possible to indicate (a part of) the contributions of that political theory for the improvement of the democratic quality of the rule of law

${ }^{1}$ Agradezco al Prof. Dr. A. Damiani por sus valiosos comentarios críticos a este trabajo. También he discutido con el Prof. Dr. D. Michelini las tesis aquí sostenidas. $\mathrm{Al}$ evaluador anónimo agradezco las sugerencias señaladas a la primera versión de este artículo, que han contribuido al mejoramiento de su calidad conceptual.

${ }^{*}$ Doctor en Filosofía (Universidad de Buenos Aires), Investigador Asistente en la Carrera del Investigador Científico (CONICET), Profesor e Investigador de la Facultad de Ciencias Jurídicas y Sociales (UNL). Miembro del cuerpo Docente del Doctorado en Derecho de dicha Facultad. Director de proyectos de investigación de la UNL y del Fondo para la Investigación Científica de la Agencia Nacional de Promoción Científica y Técnica. Dirección electrónica: santiagoprono@hotmail.com 
Keywords: Pragmatic Turn Discourse, Deliberative Democracy, Rational Reconstruction, Critique.

\section{Introducción.}

El pragmatismo destaca la naturaleza dinámica y constructiva de la racionalidad, y no separa la práctica de la teoría: ésta es concebida como una dimensión inseparable de la praxis. El saber, considerado en términos generales, es resultado de una construcción cultural colectiva, es lo que se hace en el curso de la investigación en el sentido de un esfuerzo comunitario, no el producto de una meditación individual y solitaria como la cartesiana. Así, el concepto de acción se encuentra en el centro del análisis y la explicación pragmatistas de la racionalidad. ${ }^{2}$ En este marco, el denominado "giro pragmático" de la Filosofía contemporánea implica un cambio en los presupuestos del conocimiento y de la acción imperantes en la filosofía desde, al menos, la Modernidad. Se trata de un movimiento filosófico que comporta una serie de tesis, las cuales en general pueden caracterizarse por su sentido crítico y de ruptura respecto de la cosmovisión anterior que, paralelamente al hundimiento de la garantía de certeza en el conocimiento dada por el sujeto moderno y por la realidad como instancia predeterminada y autosuficiente, reconoce al mismo tiempo el carácter intersubjetivo del pensamiento y del lenguaje. ${ }^{3}$ Estos son considerados como resultados recíprocamente

2 Cfr. Cabanchik, Samuel,Introducciones a la filosofía, Barcelona, Gedisa, 2000, p. 131 ss., e "Introducción", en Cabanchik, Samuel, Penelas, Federico, Tozzi, Verónica (eds.), El giro pragmático en filosofía, Barcelona, Gedisa, 2003, p. 13 ss.

3 En efecto, mientras que para Dewey la formulación, y posible solución, de un problema genuino implica siempre un proceso progresivo de investigación en el que intervienen diversas partes para intentar lograr dicho objetivo (Dewey, John, La buisqueda de la certeza. Un estudio de la relación entre el conocimiento y la acción (1929), Méjico, FCE, 1952, pp. 119 ss., esp. 127 ss.), para Peirce el proceso de interpretación intersubjetiva de una comunidad de intérpretes desarrollado a largo plazo permite determinar el sentido y significado de los signos, y en donde el conocimiento implica esencialmente la noción de comunidad (Peirce, Charles Sanders, The Essential Peirce. Selected Philosophical Writings. Volume 2 (1893-1913), Indiana, Indiana University Press, 
presupuestos en todo procedimiento encaminado a la solución de problemas teóricos o prácticos.

En el ámbito de la teoría política contemporánea, para los llamados "filósofos posmodernos", el pragmatismo comporta posturas deflacionistas con respecto al conocimiento, y en donde las pretensiones de universalidad de la razón, al igual que en su momento la religión, serán substituidas por algo mejor. 4 En este contexto el posicionamiento adoptado en relación con la legitimidad democrática, y con valores políticos como la "igualdad", es que no hay respuestas generales, universalmente válidas, sino sólo dudas particulares que la gente tiene acerca de las prácticas políticas particulares. ${ }^{5}$ Este posicionamiento obedece a un uso pragmático de la racionalidad, pero entendido éste en el sentido de la conveniencia, del cálculo estratégico en términos de costo-beneficio como definitorio del curso de acción más eficaz (o ventajoso) para el objetivo que se pretende alcanzar. Unabordaje de este tipo es el que plantea I. Shapiro cuando analiza el problema de la definición de los intereses básicos de un ordenamiento político, puesto que según su "aproximación pragmática" al mismo conviene adoptar una posición consistente en describir a tales intereses básicos "de un modo comparativamente débil para minimizar su carácter controvertido", y así

1998, pp. 255-256. Véase también de este autor Collected Papers, vol. I-VI, 5.289, cit. en Apel, Karl-Otto,Transformation der Philosophie. Band 2: Das A priori der Kommunikationsgemeinschaft (1973) (en adelante TPh), Frankfurt, Suhrkamp, 6. Aufl. 1999, p. 169).

${ }^{4}$ Cfr. Rorty, Richard, Consecuencias del pragmatismo, Madrid, Tecnos, 1996, pp. 19-59, esp. 54. Un ejemplo de este punto de vista en el ámbito del realismo puede apreciarse, por ejemplo, en N. Goodman y su obra Maneras de hacer mundos (1978); y en lo relativo al ámbito epistemológico, con claras implicancias socio-históricas, en Thomas Khun cuando analiza La estructura de las revoluciones cientificas (1962). En confrontación con este tipo de posturas, véase Bouveresse, Jacques, El filósofo entre los autófagos (1984), Méjico, FCE, 1989, p. 255.

5 Cfr. MacGilvray, Eric, "Democratic Doubts: Pragmatism and the Epistemic Defense of Democracy", en The Journal of Political Philosophy, vol. 22, No 1, 2014, pp. 117-118, 120. 
evitar que la definición en cuestión "se vuelva más conflictiva", dificultando la obtención de consensos. ${ }^{6}$

Ahora bien, y teniendo en cuenta aquí el señalamiento de K.-O. Apel, el problema con este tipo de posiciones pragmáticas aplicadas (en este caso) al ámbito de lo político, es que en su pretensión de desconocimiento del carácter universal, no meramente utilitarista y de alcance restringido de la racionalidad, no pueden renunciar a exponer sus tesis contra la posibilidad o la necesidad de las pretensiones universales de validez mediante el recurso a la intelección filosófica de la validez universal de argumentos, porque de otro modo ellas no podrían expresarse y resultarían inadvertidas. ${ }^{7}$ Por esta razón, y dejando de lado estas posiciones críticas, auto-contradictorias en el planteo de sus argumentos, el presente trabajo se propone alcanzar dos objetivos teóricos: el primero se orienta a explicitar y analizar la recepción del giro pragmático en la democracia deliberativa de Jürgen Habermas teniendo en cuenta para esto su concepción de la Filosofía del lenguaje, que no desconoce, sino que presupone, las pretensiones universales de validez del discurso filosófico para la fundamentación racional de decisiones políticas y normas jurídicas (adoptadas en contextos democrático-deliberativos); sobre esta base, el segundo objetivo pretende justificar uno de los aportes de esta teoría para el mejoramiento de la calidad democrática del estado de derecho a partir de la evaluación crítica del desempeño de sus instituciones.

La siguiente es la estructura expositiva de argumentos propuesta para analizar el tema y alcanzar los objetivos señalados. En la primera parte del trabajo se exponen algunos estudios contemporáneos sobre el pragmatismo en relación con la teoría política (1). A continuación, se analiza el giro pragmático de la Filosofía contemporánea y el modo en que el mismo se expresa en los presupuestos filosóficos acerca del lenguaje y de la acción comunicativa de la teoría del discurso (2). Este análisis no sólo permite explicitar el marco conceptual de la democracia deliberativa de J. Habermas como receptora de (parte de los presupuestos) del pragmatismo (3), sino

${ }^{6}$ Cfr. Shapiro, Ian,La teoría de la democracia en el mundo real, Madrid, Marcial Pons, 2011, pp. 272, 273.

${ }_{7}$ Cfr. Apel, Karl-Otto,Semiótica filosófica (en adelante SF), Buenos Aires, 1994, p. 154 ss. (esp. 162-163). 
también señalar (y justificar) una de las contribuciones de esta teoría política que, teniendo en cuenta su sentido reconstructivo, es posible realizar para el mejoramiento del desempeño institucional del estado democrático de derecho: se trata de la identificación de las falencias de dicho desempeño, y la consecuente posibilidad de consolidar teóricamente la justificación del derecho ciudadano al ejercicio de la crítica política respecto del gobierno, que es uno de los derechos esenciales y presupuestos en todo ordenamiento democrático(4). Las reflexiones finales sólo estriban en una breve exposición acerca de cómo se relacionan los argumentos presentados con las conclusiones alcanzadas (5).

\section{Pragmatismo y teoría política.}

La recepción del pragmatismo en la teoría política ha sido analizada por numerosos autores con distintos enfoques sobre la democracia deliberativa. A. Honneth, ${ }^{8}$ H. Richardson, ${ }^{9}$ J. Bohman, ${ }^{10} \mathrm{Ch}$. Misak, ${ }^{11} \mathrm{~J}$. Tully, ${ }^{12}$ M. Fentestein, ${ }^{13}$ y A. Kadlec, ${ }^{14}$ son sólo algunos ejemplos de cómo varía la conexión imputada en relación con la concepción adoptada de la deliberación en política, lo cual por supuesto expresa que no hay un entendimiento unitario del tema. En el ámbito de la teoría política

\footnotetext{
${ }^{8}$ Honneth, Axel, "Democracy as Reflexive Cooperation: John Dewey and the Theory of Democracy Today", en Political Theory, vol. 26, No 6, 1998, pp. 763-783.

9 Richardson, Henry, "Truth and Ends in Dewey's Pragmatism", Canadian Journal of Philosophy, vol. 28 (No especial "Pragmatism"), 1998, pp. 109-147.

10 Bohman, James, "Democracy as Inquiry, Inquiry as Democratic: Pragmatism, Social Science and the Cognitive Division of Labour", en American Journal of Political Science, vol. 43, No 2, 1999, pp. 590-607.

11 Misak, Cheryl, Truth, Morality and Politics: Pragmatism and Deliberation, Londres, Routledge, 2000.

12 Tully, James, "The Unfreedom of the Moderns in Comparation of their Ideals of Constitutional Democracy", en Modern Law Review, vol. 65, No 2, 2002, pp. 204-228.

13 Festenstein, Matthew, "Deliberative Democracy and two Models of Pragmatism" (en adelante DDMP), en European Journal of Social Theory, 7, vol. 3, 2004, pp. 291-306.

${ }^{14}$ Kadlec, Alison, "Critical Pragmatism and Deliberative Democracy", en Theoria: $A$ Journal of Social and Political Theory, No 117, 2008, pp. 54-80.
} 
contemporánea el pragmatismo se relaciona, por ejemplo, con posturas de corte liberal, como es el caso del liberalismo de tipo constructivista, pero también kantiano, presentado por J. Rawls en su obra Liberalismo político (1993). Según Rawls, debido al hecho del pluralismo "el punto de vista liberal quita de la agenda política las cuestiones que pueden suscitar más divisiones, que pueden causar resistencias y minar las bases de la cooperación social". 15 Este tipo de razonamiento se evidencia en su ideal deliberativo de reciprocidad, en donde también parece primar un uso meramente estratégico de la racionalidad (en el sentido antes señalado) al exigir que los ciudadanos deben abstenerse de apelar a sus creencias comprehensivas (de tipo moral, filosófico, o religioso) cuando participan en el procedimiento decisorio sobre temas de justicia básica. La razón esgrimida es que, aun cuando aquellos ciudadanos crean que estén correctamente fundamentadas, tales creencias pueden dificultar la obtención de acuerdos en torno al diseño institucional de una sociedad. ${ }^{16}$ Este es precisamente el sentido de "La prioridad de la democracia sobre la filosofia" que ha postulado R. Rorty cuando describe al liberalismo político de Rawls. De hecho, afirma en términos que a su entender resultarían aceptables para el propio Rawls, que "para los propósitos de la teoría social podemos dejar de lado tópicos como la naturaleza humana a-histórica, la naturaleza de la personalidad, los motivos del comportamiento

15Rawls, John,Liberalismo político, México, FCE, 1995, p. 157. Para el análisis de este tema en este autor véase su escrito de 1980 "Kantian constructivism in moral theory. Rational and full autonomy”, en Rawls, J.,Journal of Philosophy, vol. 77, N 9, 1980, pp. 515-572, esp. pp. 517, 518.

16 Cfr. Prono, Santiago, "Democracia deliberativa e ideal de reciprocidad. Un análisis desde la teoría del discurso", en Revista Argumentos (Fortaleza, Brasil), N 13, (en prensa), 2015. Por su parte, también Robert Talisse parece vincular a Rawls con esta concepción estratégica, utilitarista de la racionalidad, señalando que en sus estudios sobre el tema los pragmatistas contemporáneos ignoran o desatienden el pensamiento rawlsiano (Cfr. Talisse, Robert, "John Rawls and American Pragmatisms", Exposición en Congreso: Rawlsian Liberalism in Context(s), University of Tennessee, Knoxville, E.E.U.U. 26-27 Febrero de 2010. (http://papers.ssrn.com/sol3/papers.cfm?abstract_id=1611859). 
o el significado de la vida humana. Los tratamos como tan irrelevantes para la política como las cuestiones acerca de la Trinidad o latransubstanciación".. ${ }^{17}$

Estos planteos acerca de la teoría política y relacionados con el pragmatismo parecen vincularse, al menos el último comentario señalado, con una concepción devaluada de la racionalidad en lo que respecta a sus pretensiones universales de validez aplicadas al ámbito de lo político. Distinto es el caso de M. Festenstein, quien, apartándose de algunas interpretaciones del pragmatismo que a su entender comportan una concepción devaluada de las exigencias cognitivas inherentes a toda teoría política, asume un compromiso con las mismas explicitando las condiciones que promueven el florecimiento humano, aquí interpretado como una actitud de investigar (inquiry). Esto implica "centrar la atención en las virtudes epistémicas como (...) constitutivas de una amplia concepción del florecimiento humano [, que redunda] en un mayor compromiso con las necesidades e intereses de los otros". 18

Una concepción del pragmatismo que, como la anterior, ya no desconoce razones de orden epistémico de alcance universal, se encuentra también en R. Talisse, quien desarrolla sus estudios a partir de la filosofía Ch. S. Peirce. Esto se evidencia cuando señala que confrontar deliberativamente con quienes se disiente "es esencial aun cuando tengamos la verdad, [porque] incluso si nuestra 'gran verdad' es verdadera, discutir solamente con quienes acordamos, sin comprometernos a interactuar con visiones opuestas, puede cambiar nuestra posición hacia una visión en la que perdamos la verdad. ${ }^{19} \mathrm{La}$ idea es que el valor epistémico de las creencias (en este caso) políticas, se fundamenta en el marco de una comunidad de intérpretes deliberantes sobre temas políticos, y que forman parte de un procedimiento que progresa de manera indefinida.

17 Rorty, Richard, “The Priority of Democracy to Philosophy" (1988), en Rorty, R., Objectivity, Relativism and Truth: Philosophical Papers Volume 1, Cambridge, Cambridge University Press, 1991, p. 180.

18DDMP, p. 302, cfr. pp. 299-302.

${ }^{19}$ Talisse, Robert,Democracy after Liberalism. Pragmatism and deliberative politics, New York, Routledge, 2005, p. 118, cfr. pp. 119-120. 
En esta línea argumentativa, y criticando toda recepción de los presupuestos del pragmatismo que implique una devaluación de la racionalidad y de sus pretensiones universales de validez, expresadas por ejemplo en conceptos como normatividad, o legitimidad, E. Erman y N. Möller analizan el tema interrogándose acerca de "Qué es posible esperar del giro pragmático en la teoría política". ${ }^{20}$ Estos autores objetan la interpretación política que realiza Ch. Mouffe de la multiplicidad de los juegos de lenguaje propuesta por Wittgenstein en sus Investigaciones filosóficas, y la que lleva a cabo T. Fossen en su análisis de la obra de R. Brandom, Making it Explicit. En opinión de aquellos autores, tales interpretaciones resultan controversiales, en el primer caso, debido a que la multiplicidad de juegos de lenguaje, expresada en sociedades diversas y divergentes, no impide establecer, y fundamentar, principios políticos de carácter normativo, entre otras razones, porque lo contrario "[implica] una devaluación de los caracteres semánticos, ontológicos y epistémicos que dificulta toda pretensión de dotar de respaldo normativo a la teoría política". ${ }^{21}$ En lo que respecta a T. Fossen, se objeta también las implicancias que de la lectura de este autor (sobre la obra de Brandom) se siguen para la legitimidad política. Erman y Möller señalan que tal interpretación resulta conceptualmente problemática para la teoría política

20 Erman, Eva y Möller, Niklas; "What not to expect from the pragmatic turn in political theory" (en adelante EJPT), en European Journal of Political Theorys publicado online el 5 de junio de 2014, en http://ept.sagepub.com/content/early/2014/06/04/1474885114537635, pp. 1-20.

21 Cfr. EJPT, pp. 7-8, cfr. pp. 12, 13. En efecto, Mouffe, e inspirándose en Wittgenstein, concibe a la política democrática como "receptora de la multiplicidad de voces que albergan las sociedades plurales contemporáneas, y de la complejidad de sus estructuras de poder" (Mouffe, Chantal,La paradoja democrática (en adelante PD), Barcelona, Gedisa, 2003, p. 118). Así, su concepción agonista de lo político es dependiente de contextos particulares, y refractaria de toda concepción universalista de la moralidad que permita establecer principios normativos en el ámbito político (cfr. PD, p. 78; cfr. Mouffe, Chantal,En torno a lo político, Buenos Aires, FCE, 2007, p. 129). Un análisis crítico de esta concepción de lo político respecto de la deliberación democrática se encuentra en Prono, Santiago; "La democracia deliberativa y el problema de su implementación práctica. El debate consenso vs. conflicto", en Universitas. Revista de filosofia, derecho y política, Universidad Carlos III (Madrid, España), No 12, 2010, pp. 113-134. 
debido a que en ella "la pretensión de legitimidad no puede ser determinada con certeza, y [por esto] falla en sugerir cualquier tipo de teoría normativa de la legitimidad". ${ }^{22}$ Esta crítica al planteo de Fossen se fundamenta en el hecho de que Brandom en verdad no sólo que no niega, sino que más bien sugiere la posibilidad de establecer, por ejemplo, pretensiones universales de corrección normativa haciendo explícito los presupuestos de la práctica discursiva, y a partir de cuyo reconocimiento es posible fundamentar la pretensión de legitimidad de un ordenamiento político-democrático. ${ }^{23}$

Aun cuando reconocen que el pragmatismo de autores como Wittgenstein o Brandom pueda tematizarse desde el punto de vista de la teoría política, según Erman y Möller estas interpretaciones (de Mouffe o de Fossen) sobre el pragmatismo filosófico realizadas desde la teoría política contemporánea resultan conceptualmente problemáticas, dado que en ellas se plantea un déficit respecto de presupuestos centrales de la teoría política, como la normatividad y la legitimidad democrática.

Ahora bien, teniendo en cuenta estos, pero también los anteriores autores presentados, cuyos planteos se relacionan de diversas maneras con diversas concepciones del pragmatismo y su correspondiente aplicación al ámbito de la política democrática, ¿qué recepción de dicha tradición de pensamiento filosófico es posible establecer en el marco teórico de la democracia deliberativa?, ¿cuál es la relevancia conceptual del giro pragmático y de su concepción de la racionalidad que resulta teóricamente relevante en el contexto de la Filosofía del lenguaje presupuesta por esta teoría política?, y ¿qué aporte a partir de allí es posible señalar para el mejoramiento del desempeño institucional del estado democrático de derecho? A fin de responder a estos interrogantes es necesario comenzar explicitando los fundamentos de la teoría del discurso y su interpretación del giro pragmático

22 EJPT, pp. 14, 15.

23 Efectivamente, según Brandom, a partir del análisis reconstructivo orientado a explicitar los presupuestos del lenguaje comunicativo es posible establecer reglas y principios normativos de alcance general (cfr. Brandom, Robert,Making it Explicit. Reasoning, Representing and Discursive Commitment (en adelante ME), Cambridge, Massachusetts, Harvard University Press, 1994, p. 779). 
de la Filosofía contemporánea, porque constituye la base filosófica sobre la cual se apoya la democracia deliberativa de J. Habermas.

\section{El giro pragmático y la teoría del discurso como fundamentos filosóficos de la democracia deliberativa.}

El giro pragmático de la Filosofía contemporánea, aquí interpretado en el marco de la Filosofía del lenguaje asumido por la teoría del discurso de Habermas y de Apel, se centra en el análisis de las acciones que los hablantes realizan cuando hacen uso del lenguaje para comunicarse entre sí, y explicita los presupuestos reconocidos por todo interlocutor cuando plantea un argumento orientado a la solución racional de un problema teórico o práctico. Esto supone una nueva concepción del lenguaje y la racionalidad que comienza ya a entreverse, al menos, en el lingüista alemán W. von Humboldt. Para este autor el lenguaje ya no es un sistema de signos, tampoco es un producto del pensamiento, sino una actividad ya siempre presupuesta por este último, y estrechamente relacionada con la intersubjetividad como proceso dialógico qua expresión de su dimensión comunicativo-pragmática. ${ }^{24}$ Se trata de una concepción que, podría decirse, contribuyó a la configuración del marco conceptual dentro del cual comenzó a gestarse el mencionado giro (también lingüístico) de la filosofía del siglo XX,25 el cual se constituye a partir de la confluencia de determinadas teorías filosóficas.

24 Para un análisis de las obras de este autor véase de Andreas Flitner y Klaus Giel, Schriften zur Sprachphilosophie, (1979, vol. 3), cit. en Lafont, Cristina,La razón como lenguaje. Una revisión del 'Giro lingüístico' en la filosofía del lenguaje alemana, Madrid, Visor, 1993, pp. 31 ss. Citas y comentarios de las obras de Wilhelm von Humboldt sobre este tema también están en Valverde, G., Guillermo de Humboldt y la Filosofía del Lenguaje, Madrid, Gredos, 1955, p. 30 ss.

25 De hecho esto es algo que el mismo Apel parece reconocer cuando tematiza su punto de vista respecto de la acción comunicativa por medio del lenguaje (cfr. Apel, Karl-Otto, Auseinandersetzungen in Erprobung des transzendentalpragmatischen Ansatzes, Frankfurt, Suhrkamp, 1998, pp. 9 ss). Se trata de un reconocimiento que también se encuentra en Gadamer, Hans-Georg,Verdad y método Salamanca, Sígueme, 1977, p. 146; en Habermas, Jürgen, $W$ ahrbeit und Rechtfertigung (en adelante WR), Frankfurt, 
Entre estas teorías se encuentra la de los juegos de lenguaje entretejidos con las diversas formas de vida proveniente del segundo Wittgenstein, en cuyas Investigaciones filosóficas ya no cabe hablar de un "lenguaje privado". ${ }^{26}$ Para este filósofo la estructura, el funcionamiento, y el significado del lenguaje no son independientes del "uso" que del mismo hagan los interlocutores en el marco de sus diversas prácticas comunicativas. ${ }^{27}$ En este sentido también puede mencionarse la teoría de los actos de habla de J. L. Austin y J. Searle, para quienes tampoco corresponde analizar el problema de la significatividad del lenguaje solamente en términos de su referencialidad, desconociendo las acciones que comportan su esencial carácter intersubjetivo-comunicativo. ${ }^{28}$ Por último también cabe señalar la semiótica pragmaticista que procede de Ch. S. Peirce, que trasciende la semántica referencial del positivismo lógico poniendo especial énfasis en el carácter intersubjetivo del lenguaje y la racionalidad. ${ }^{29}$

En todos estos enfoques teóricos se evidencia una ampliación del criterio de significado semántico-referencial de la verificabilidad o refutabilidad de las proposiciones, asignando una prioridad (no excluyente) a la dimensión comunicativa e intersubjetiva del lenguaje y la racionalidad, y

Suhrkamp, 1999, pp. 67 ss); y, recientemente, también en Hösle, Vittorio,Eine kurze Geschichte der deutschen Philosophie, München, C. H. Beck, 2013, pp. 114-116.

${ }^{26}$ Cfr. Wittgenstein, Ludwig,Investigaciones filosóficas (en adelante IF), Barcelona, Crítica, 1988, $\mathbb{} 43$.

27 Cfr. IF $\$ 3, \$ 26, \$ 37$.

28 Cfr. Austin, John Langshaw,Cómo hacer cosas con palabras, Barcelona, Paidós, 1982, pp.41, 43, 54; Searle, John, Actos de habla, Madrid, Cátedra, 1994, pp. 25-26 (un cambio de postura de este último en favor de posiciones relacionadas con la filosofía de la mente y el punto de vista solipsista, anterior al giro pragmático-lingüístico de la filosofia contemporánea, se evidencia en sus obras Intentionality: An Essay in the Philosophy of Mind, 1983, y Mind: a Brief Introduction, Oxford, O.U.P., 2004).

${ }^{29}$ Cfr. Peirce, Charles Sanders, The Essential Peirce. Selected Philosophical Writings. Volume 2 (1893-1913). Indiana. Indiana University Press. 1998, pp. 202, 255-256, 274-275, 323, 324.J.O. Urmson considera a Peirce como uno de los fundadores del pragmatismo, en Urmson, James Opie, Enciclopedia concisa de Filosofía y filósofos, Madrid, Cátedra, 1982, p. 324. Una valoración similar realiza Karl-Otto, Apel (cfr. Apel, TPh, p. 173, SF, pp. 165 ss.). 
anulando el paradigma subjetivista de la conciencia, propio de la Filosofía Moderna. Como consecuencia de este desplazamiento se subraya ahora la centralidad de la pragmática del lenguaje, representada por el referido análisis de las acciones que los hablantes realizan cuando hacen uso del mismo, lo cual constituye un criterio ineludible para la constitución, y comprensión, del significado. El sentido reconstructivo inherente a esta reflexión sobre los presupuestos de la dimensión pragmática del lenguaje, se evidencia en la pragmática universal del lenguaje de J. Habermas. Ya en su escrito de 1976 (“¿Qué significa pragmática universal?”) afirma el filósofo que la expresión o manifestación de un enunciado satisface tres tipos de pretensiones de validez, según las cuales los oyentes lo consideran verdadero en la medida en que refleje algo perteneciente al mundo, veraz si expresa las intenciones del hablante, y normativamente correcto si afecta expectativas socialmente reconocidas. La pragmática universal del lenguaje analiza entonces la capacidad de comunicación del hablante para hacerse entenderpor medio de determinados actos de habla, los cuales presuponen estas pretensiones de validez señaladas (aunque por supuesto siempre es una sola la que tiene preeminencia de acuerdo al tema de que se trate). ${ }^{30}$

La explicitación de los presupuestos de la acción comunicativa, permite evidenciar que toda expresión lingüística, expresada como pretensión de validez respecto de cualquier tema, implica ya siempre el reconocimiento de una cierta comunidad de comunicación como destinataria (real o virtual) de tales actos de habla, y de cuyo reconocimiento depende la validez de tales manifestaciones. ${ }^{31}$ Ahora bien, es en este marco que Habermas establece (y

${ }^{30}$ Cfr. Habermas, Jürgen, “¿Qué significa pragmática universal?” (1976) (en adelante PU), en Habermas, Jürgen, Teoría de la acción comunicativa. Complementos y estudios previos, Madrid, Cátedra, 1997, pp. 328-329; TPh, p. 411, SF, p. 315, y Apel, Karl-Otto, Semiótica trascendental y filosofía primera, Madrid, Síntesis, 2002, p. 175.

31 En efecto, el sentido reconstructivo de la pragmática universal de Habermas da cuenta de la base de validez del habla y, siguiendo a Apel, permite explicitar lo que todo hablante, que plantee pretensiones de validez mediante un discurso argumentativo, necesariamente ya siempre ha de presuponer (y aceptar) como condiciones normativas de la posibilidad del entendimiento intersubjetivo: cfr. PU, pp. 299-300,TPh., pp. 358 ss., esp. 399-401, 406, 411; “Diskursethik als Ethik der Mit-Verantwortung vor den Sachzwängen der Politik, des Rechts und der 
Apel, aunque con reservas, reconoce) el principio del discurso como criterio ineludible de fundamentación racional: "válidas son aquellas normas de acción, y sólo aquellas, a las que todos los posibles involucrados puedan dar su asentimiento en tanto que participantes en discursivos racionales". 32 Para este principio, fundamental en la teoría del discurso de Habermas y de Apel, y por supuesto también en la teoría de la democracia deliberativa, la fundamentación racional de pretensiones de validez planteadas en el marco de un uso comunicativo del lenguaje, implica la necesidad de apelar a discursos argumentativos, públicamente expresados, que puedan alcanzar un reconocimiento intersubjetivo a partir de las razones en que se fundamentan. ${ }^{33}$

Así, frente a las múltiples versiones del giro pragmático de la Filosofía contemporánea, la pragmática universal de Habermas se presenta entonces como una interpretación del mismo que toma en cuenta la dimensión

Marktwirtschaft", in Apel, Karl-Otto, Burckhart, Holger, Prinzip Mit-Verantwortung. Grundlage für Ethik und Pädagogik, Würzburg, 2001, pp. 71-73; Damiani, A., "Teoría de

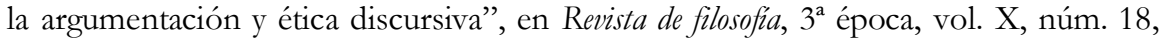
1997, p. 214, "Comunidad, realidad y pragmatismo", en Tópicos. Revista de filosofía de Santa Fe, No 20, 2010, p. 137, "Acción, interpretación y discurso", en Michelini, Dorando, Prono, Santiago, Kalpokas, Daniel (eds.), Ética del discurso. Su significación para la filosofía práctica y el diálogo intercultural, Río Cuarto, Icala, pp. 18-20).

${ }^{32}$ Habermas, Jürgen,Faktizität und Geltung. Beiträge zur Diskurstheorie des Rechts und des demokratischen Rechtstaats (en adelante FG), Frankfurt, Suhrkamp, p. 138, cfr. FG 196; Alexy, Robert,La institucionalización de la justicia, Granada, Comares, pp. 61-62. Apel señala la insuficiencia de este principio dado que Habermas ya no aceptaría los presupuestos morales que resultan inherentes al ejercicio de la racionalidad mediante el planteo de argumentos con pretensión de sentido. En opinión de Apel esto conduce a la disolución de la teoría ética del discurso, que ambos desarrollaran en conjunto desde comienzos de 1970 (cfr. FG, pp. 137, 139). Las críticas de Apel están en Apel, Karl-Otto,Diskurs und Verantwortung, Frankfurt, Suhrkamp, 1998, pp. 651 ss, esp. pp. 733-742.

33 Cfr. Habermas, Jürgen,Teoría de la acción comunicativa. II: Crítica de la razón funcionalista(en adelante TkH)(1981), Madrid, Taurus, 1987, pp. 323-324. Naturalmente, esto no implica desconocer el carácter falible de las decisiones que se obtengan como resultado de este tipo de procedimiento intersubjetivo de deliberación racional, pero sí que este último es el criterio de validez de las mismas. 
esencialmente dialógica y comunicativa de la razón a partir de descubrir, o explicitar reconstructivamente, la validez universal de los presupuestos normativo-morales implícitos en la facticidad de la argumentación. ${ }^{34}$

Llegados a este punto son tres entonces las características conceptualmente relevantes de la teoría del discurso que pueden identificarse con el mencionado giro (lingüístico-) pragmático de la Filosofía contemporánea: la superación del solipsismo metódico, es decir, la superación del paradigma moderno de la conciencia; que presupone el carácter intersubjetivo de la racionalidad comunicativa, y que, por último, se fundamenta a partir del procedimiento reconstructivo de los presupuestos inherentes a los actos de habla implícita o explícitamente reconocidos por todo interlocutor discursivo. Se trata pues de características que también resultan conceptualmente relevantes en el marco teórico de la democracia deliberativa, y en base a las cuales es posible, luego, señalar sus aportes al estado democrático de derecho.

\section{Giro pragmático y Filosofía política contemporánea: la teoría de la democracia deliberativa.}

El anterior es el marco conceptual de la teoría del discurso de Habermas, también presupuesto por su concepción de la democracia

\footnotetext{
34 Según D. Böhler la reconstrucción filosófica comporta un sentido cognitivo y normativo aplicado al conocimiento práctico que permite entender, para así juzgar y fundamentar, las acciones y las diversas orientaciones para la acción (cfr. Böhler, Dietrich,Rekonstruktive Pragmatik. Von der Bewusstseinsphilosophie zur Kommunikationsreflexion: Neubegründung der praktischen Wissenschaften und Philosophie, Frankfurt, Suhrkamp, 1985, pp. 242, 243). Dicho procedimiento permite identificar criterios normativos, en el sentido que (aunque desde otra perspectiva filosófica, también) señala R. Brandom, cuando refiere a la explicitación del saber inherente al uso comunicativo del lenguaje (cfr. ME, p. 77). Esta tarea de reconstrucción racional puede leerse a la luz de la clásica distinción de G. Ryle (planteada en su obra de 1949, The Concept of Mind) entre know how y know that, es decir, entre el saber cómo, y el saber que, dado que permite diferenciar entre la capacidad de un sujeto para formar oraciones y comunicarse con otros, por un lado, y las reglas y presupuestos que lo hacen posible, por el otro. Para un análisis de este tema desde la filosofía analítica véase Strawson, Peter, Análisis y metafísica, Barcelona, Paidós, 1992, pp. 41 ss.
} 
deliberativa. Así, esta teoría política reconoce el carácter esencialmente comunicativo $e$ intersubjetivo de la racionalidad, lo cual implica, consecuentemente, una superación del solipsismo metódico, propio de la Filosofía moderna. Se trata de una interpretación (normativa) de lo político como deliberación, en el sentido de un intercambio de opiniones antitéticas que, al menos en principio, tienen que confrontarse en términos de argumentos para intentar llegar a la mejor decisión posible y lograr acuerdos racionalmente motivados. La teoría política expuesta por Habermas (aunque no exclusivamente) en su principal obra filosófico-político-jurídica, Facticidad y validez. (1992), adopta entonces un carácter normativo y procedimental (pero también substantivo) cuyo principio básico es el (ya citado) principio del discurso. Dicho principio, que ocupa un lugar central en las teorías del derecho y de la moral de este filósofo (y también de Apel), implica, como se señaló, un procedimiento para el tratamiento de cuestiones de validez o de justicia, según el cual las decisiones (en este caso) políticas son legítimas, y pueden ser reconocidas como tales, en la medida en que se adopten a partir de una deliberación democrática, entendida como un conjunto de acciones lingǘsticas consistente en la realización de un intercambio público de razones en busca de un consenso. Habermas concibe a este principio como un uso del lenguaje que presupone exigentes compromisos asumidos por el hablante, en el sentido de que (1) todo hablante puede participar en dicho proceso, (2) todo participante puede expresar sus opiniones, presentar nuevos argumentos, o criticar los ya planteados, y (3) nadie puede ser privado, mediante coacción dentro o fuera del discurso, de ejercer los derechos establecidos en (1) y (2). ${ }^{35}$ De acuerdo con estas exigencias discursivas, todo interlocutor tiene el mismo derecho de participar de dicho procedimiento, y el mismo deber de justificar sus opiniones atendiendo exclusivamente a la fuerza que sólo los mejores argumentos pueden ejercer, y cuya característica distintiva es la simetría, "puesto que quienes participan de tal procedimiento no asumen roles diferenciados dependientes de funciones específicas, sino que todos asumen

35 Cfr. Habermas, Jürgen,Moralbewusstsein und kommunikatives Handeln, Frankfurt, Suhrkamp, 1983, p. 103, FG, pp. 155-157; Alexy, Robert,El concepto y la validez del derecho, Barcelona, Gedisa, 2004, pp. 137-138. 
un mismo rol: el de interlocutor discursivo". ${ }^{6}$ En el marco teórico de la democracia deliberativa estas exigencias del discurso argumentativo, que son implícitamente presupuestas y formalmente reconocidas en el ordenamiento institucional de un estado democrático de derecho, constituyen reglas procedimentales que justifican el estatus epistémico y la validez normativa de las decisiones adoptadas conforme a esas reglas.

Así entendida, la teoría habermasiana de la democracia deliberativa implica un proceso de discusión y confirmación de voluntades políticas en el que aparece de manera explícita la estructura intersubjetiva de la argumentación (que presupone el reconocimiento de derechos), y el carácter comunicativo de la autolegislación: mientras que la estructura intersubjetiva hace posible la conexión interna entre soberanía popular y derechos del hombre, porque en el sistema de derechos se recogen las condiciones bajo las que puede institucionalizarse jurídicamente la producción políticamente autónoma, el carácter comunicativo de la autolegislación sirve como mediación discursiva entre las orientaciones de los ciudadanos hacia el bien común y las diferentes constelaciones sociales de intereses de personas privadas, es decir, representa la igualdad jurídica normativa del derecho moderno, lo cual es condición de legitimidad de las normas aceptadas por igual por todos los interesados.

$\mathrm{Al}$ igual que en la teoría del discurso, en la democracia deliberativa el diálogo argumentativo denominado "discurso" constituye un medio fundamental e ineludible para (intentar) conocer y evaluar racionalmente las acciones y las instituciones humanas, y por lo tanto también para juzgar y legitimar las pretensiones universales de validez planteadas por los distintos actores que conforman un proceso decisorio. ${ }^{37}$

${ }^{36}$ Damiani, Alberto, "Acción y racionalidad en la filosofía trascendental: del giro copernicano al giro lingüístico-pragmático” (en adelante AR), en Michelini, Dorando, Neuman Soto, Hugo, De Miguel, Raúl (eds.), Río Cuarto, Ediciones del Icala, 2011, pp. 37-38. Cfr. Maliandi, Ricardo,Ética: dilemas y convergencias(en adelante EDC), Buenos Aires, Biblos, 2006, p. 231 ss.; FG, p. 141. Para un análisis del principio de "simetría" aquí presupuesto, véase también AR p. 38.

37 Cfr. FG, pp. 141-142, WR, p. 253, Habermas, Jürgen; "Vom pragmatischen, ethischen und moralischen Gebrauch der praktischen Vernunft" (1991), en 
Finalmente, la teoría de la democracia deliberativa presupone también (como la teoría del discurso) un procedimiento reconstructivo. En efecto, y desde un punto de vista normativo, cabe destacar que la democracia deliberativa no se limita a describir cómo es la realidad, o cómo se toman efectivamente las decisiones (aunque esto es algo que sin dudas tiene en cuenta), sino que en base a dicho procedimiento identifica los presupuestos del discurso argumentativo, pero también de las prácticas políticas concretas. Así, el método reconstructivo de esta teoría señala (o descubre) las presuposiciones normativas que hay que reconocer como criterio de posibilidad y de validez de las decisiones políticas adoptadas en contextos democráticos, pretendiendo combinar así el punto de vista universal y el socio-histórico. ${ }^{38}$

La teoría habermasiana de la democracia deliberativa es parte entonces de una concepción más amplia de la filosofía práctica que comprende una teoría de la acción social y de la racionalidad (política), cuyo núcleo está en el concepto de acción comunicativa y en la pragmática universal del lenguaje, un núcleo que se fundamenta a partir del procedimiento reconstructivo de las presuposiciones operantes en la interacción comunicativa argumentativamente mediada. ${ }^{39}$ Esta explicitación del saber implícito en la acción consistente en plantear un discurso argumentativo, comporta un procedimiento que descubre los presupuestos inherentes al funcionamiento de la democracia contemporánea como sus propias condiciones de posibilidad y de legitimidad, y a partir de los cuales se fundamentan reglas o principios normativos (en el sentido de la cita de Brandom antes señalada).40

Habermas, J., Philosophische Texte (Bd. 3: Diskursethik), Frankfurt, Suhrkamp, 2009, pp. 366-367. Para un análisis del valor epistémico de este tipo de procedimiento decisorio en la democracia deliberativa, véase Nino, Carlos Santiago,La constitución de la democracia deliberativa, Barcelona, Gedisa, 2003, pp. 166-167.

${ }^{38}$ Cfr. TkH, pp. 323-324; FG, pp. 359-360, 390 ss.

39 Cfr. De Zan, Julio,La vieja y la nueva política. Libertad, Poder y Discurso II (en adelante LPD), Buenos Aires, UNSAM, 2013, pp. 245-246.

${ }^{40}$ En sección 1 , nota 23. 
Tanto si se la considera en términos generales, como si se indaga en sus presupuestos teóricos básicos, esta teoría política toma como punto de apoyo para fundamentar sus pretensiones de validez el principio del discurso argumentativo, que se fundamenta a su vez en el marco del giro pragmático de la Filosofía contemporánea al subrayar reconstructivamente el carácter, ya no meramente semántico-referencial, sino fundamentalmente pragmático y comunicativo del lenguaje y de la racionalidad para el procedimiento de fundamentación intersubjetiva de pretensiones de validez, orientadas (en este caso) a la definición, mediante deliberación discursiva y democrática, de decisiones políticas y normas jurídicas. A diferencia de planteos teóricopolíticos que, de acuerdo por ejemplo con el análisis de Erman y Möller, realizan una cuestionable recepción conceptual del pragmatismo, la democracia deliberativa de Habermas constituye una de las derivaciones teóricas que en el plano político-normativo se siguen del mencionado giro pragmático y sus correspondientes implicancias epistemológicas, presupuestas por el principio fundamental de esta teoría política, que no es el principio de la mayoría, sino del discurso.

Sobre esta base (ahora sí ya) es posible explicitar, y justificar, uno de los aportes de la democracia deliberativa al estado democrático de derecho.

\section{Democracia deliberativa y análisis crítico-reconstructivo: aportes para la evaluación del desempeño institucional de la democracia.}

Se explicitó antes (y se reiteró en el último párrafo de la sección anterior) que son tres las características conceptualmente relevantes del giro pragmático que se evidencian en el marco de la teoría del discurso, y que resultan inherentes a la democracia deliberativa (que presupone dicha teoría): el carácter intersubjetivo de la racionalidad comunicativa y la consecuente superación del solipsismo metódico, los cuales se fundamentan en el procedimiento reconstructivo de los presupuestos inherentes al planteo de todo discurso argumentativo. Pues bien, cabe destacar que este método reconstructivo de la democracia deliberativa fundamenta (parte de) sus aportes al estado de derecho, porque su implementación en el ámbito político para el análisis de los procesos justificatorios y sus correspondientes decisiones que en el mismo se adopten, contribuye a la identificación de las falencias inherentes al desempeño 
práctico de las instituciones democráticas. La idea es que a partir de esta identificación es posible justificar, y llegado el caso respaldar judicialmente, el derecho a la crítica por parte de los ciudadanos respecto de las acciones del gobierno, lo cual constituye un punto de partida para todo intento de mejorar la calidad democrática de las instituciones del estado de derecho, y ello en la medida en que éstas efectivamente se abran a las demandas y los aportes provenientes de los espacios informales de la opinión pública y la sociedad civil, porque son los ámbitos en los que se genera el poder comunicativo que otorga sustento y legitimidad al poder administrativo del gobierno.

Las condiciones normativas inherentes al discurso argumentativo (presupuesto por la política deliberativa, y por supuesto por todo ordenamiento político democrático) se expresan en los principios de simetría, que presuponen el principio de justicia, de no exclusión, no violencia, igualdad, libertad, y respeto recíproco, también susceptibles de caracterizarse como principios morales, ya siempre y necesariamente presupuestos cada vez que se plantea una pretensión universal de validez mediante un discurso argumentativo, independientemente de cuál sea el tema objeto de debate. Todos estos principios constituyen condiciones de validez propias del discurso práctico que se explicitan a partir del método reconstructivo inherente a la democracia deliberativa. Por supuesto que estas exigentes condiciones procedimentales y comunicativas señaladas, de las que también depende la legitimidad de las decisiones democráticas de los poderes políticos del Estado (incluidas aquellas que definen el contenido del derecho positivo), están muy alejadas de la realidad que muestran las investigaciones empíricas de la política. Sin embargo, el planteamiento teórico habermasiano de la política no sólo tiene en cuenta las descripciones empíricas de la ciencia política, sino que parte de ellas para sus propios análisis reconstructivos de los presupuestos operantes en el funcionamiento fáctico de las democracias. ${ }^{41}$

41 Señala Habermas que “(...) que el contenido normativo que se hace valer reconstructivamente está ya inscripto como parte de la propia facticidad de los procesos políticos observables. Una sociología de la democracia que trabaja con el método reconstructivo, tiene que seleccionar por lo tanto sus conceptos fundamentales de tal manera que pueda identificar ya en las prácticas políticas, por 
La explicitación reconstructiva de los presupuestos normativos del discurso argumentativo, también asumidos en los procedimientos decisorios de la política deliberativa, no sólo evidencia las condiciones que garantizan el valor epistémico y la legitimidad de estos procedimientos, sino que al mismo tiempo también ofrece un riguroso criterio metapolítico de orientación y de crítica del desempeño de las instituciones de la democracia cuando estas eventualmente desconozcan tales condiciones normativas. ${ }^{42}$ Es importante tener en cuenta que dicho ejercicio crítico no debe interpretarse como una instancia externa al procedimiento deliberativo de confrontación de argumentos, ubicándose en una posición superior para desde allí analizar y juzgar las prácticas institucionales: no se trata aquí de interpretar a esta teoría política en el sentido de atribuirle el papel de juez supremo de las prácticas políticas, o como poseyendo propiedades especiales "para actuar de árbitro en las sociedades democráticas a la hora de establecer o justificar normas"; mucho menos se trata de intentar fundamentar una idea de paternalismo que permita "educar al ciudadano a fin de que aprenda a colaborar y participar en la vida pública". ${ }^{3}$ Puesto que la democracia deliberativa no adopta ningún método constructivista (como es el caso de la filosofía política de J. Rawls), sino reflexivo y reconstructivo de las condiciones de validez de la argumentación, el sentido crítico que adopta esta teoría no se ubica en un nivel distinto de las prácticas habituales, sino que se justifica teniendo en cuenta este carácter reconstructivo, y por el cual puede también constituirse

distorsionados que ellos se encuentren, los aspectos y los fragmentos de una razón existente [en el sentido de una racionalidad discursiva que plantea pretensiones de validez racionalmente justificables]" (FG, p. 349). Al respecto, véase también LPD, p. 252.

42 Cfr. Michelini, Dorando, "Ética discursiva y legitimidad democrática" (1991), en Apel, Karl-Otto, Cortina, Adela, De Zan, Julio y Michelini, Dorando (eds.), Ética comunicativa y democracia, Barcelona, Crítica, 1991, pp. 334-335.

43 Cfr. A. Cortina y V. Camps, quienes confieren estas características práctico-teóricas a la ética del discurso en el campo de la política democrática, en Cortina, Adela, "Ética discursiva y democracia política", en Revista colombiana de psicología, No 2, 1993 , p. 109, y Razón comunicativa y responsabilidad solidaria, Sígueme, Salamanca, 1995, pp. 181 ss.; y Camps, Victoria., "Comunicación, democracia y conflicto", en Apel, Karl-Otto, Cortina, Adela, De Zan, Julio y Michelini, Dorando (eds.), Ética comunicativa y democracia, Barcelona, Crítica, 1991, pp. 248, 249-250, 254. 
en un punto de apoyo para el análisis y la crítica inmanente del desempeño de las instituciones del estado democrático de derecho (que Habermas conceptualiza en términos de su teoría del discurso), señalando eventuales inconsistencias de tal funcionamiento a partir del posible desconocimiento de los principios antes señalados, que son también condición de legitimidad del desempeño de dicho estado democrático.

Está claro que el riesgo que aquí hay que evitar es caer en aquellos lugares comunes de los señalamientos obvios, ya que en relación con el análisis de temas, por ejemplo, relacionados con la corrupción y la arbitrariedad de regímenes formalmente democráticos, o con la proliferación de regímenes autoritarios y dictatoriales que resultan violatorios de los derechos humanos, si bien son temas ciertamente importantes, en realidad toda teoría política (y también ética) los concebiría como problemas que hay que solucionar. ${ }^{44}$ Sin embargo, el sentido reconstructivo (del principio del discurso y por lo tanto también) de la democracia deliberativa, dado que explicita las condiciones de posibilidad de la reciprocidad discursiva, es especialmente sensible y crítico de las asimetrías injustificadas que subsisten en los procedimientos decisorios (por ejemplo, para la generación legítima del derecho), aumentando la probabilidad de que el discurso práctico resulte razonable y equitativo, y permitiendo de este modo identificar claramente las contradicciones entre dos diferentes niveles fácticos: las contradicciones entre, por un lado, aquello a lo cual las instituciones democráticas están obligadas de acuerdo a la ley establecida (incluida la Constitución Nacional), y, por el otro lado, cómo tales instituciones actúan, es decir, de qué modo éstas se desempeñan en relación con las decisiones que adoptan y los procedimientos en que se basan. Así entendido, el orden normativo, jurídica y democráticamente diseñado de un estado de derecho, además de la fundamentación mediante razones por parte de los miembros que lo componen, tiene que presuponer también la posibilidad de crítica ejercida desde los fundamentos de la teoría del discurso, en el sentido de una crítica

${ }^{44}$ Cfr. EDC, pp. 218 ss. 
hacia aquellas legitimaciones que pretenden presentar como fundamentadas decisiones políticas que en realidad no pueden justificarse. ${ }^{45}$

Esta concepción de análisis crítico-reconstructivo del desempeño institucional de la democracia, también es reconocida por Habermas cuando sostiene que el concepto de "estado de derecho con división de poderes, extrae su legitimidad de una racionalidad que garantiza la imparcialidad de los procedimientos legislativos y judiciales. [Y por ello este concepto] no representaría otra cosa [más] que un estándar crítico (ein kritischer Maßstab) para el análisis de la realidad constitucional". ${ }^{46}$ Esta es la base teórica a partir de la cual es posible, luego, diseñar y establecer criterios que sirvan como puntos de referencia fundamentales para evaluar críticamente el desempeño de las instituciones democráticas: cabe recordar que en una democracia representativa las decisiones, el control de los recursos económicos, la aplicación de la fuerza y el monopolio de la misma por parte del Estado, se delegan en personas e instituciones estatales. Casi todo lo que es importante en la vida política de una sociedad está en manos de sus representantes. Por ello es importante que los ciudadanos preserven por lo menos el derecho a la crítica hacia aquellos en quienes han delegado (casi) todo. Así, el derecho ciudadano a la protesta ( $\mathrm{y}$ a la crítica) es un derecho fundamental de todo ordenamiento político: este derecho es el fundamento para poder preservar otros derechos, ya que si este falta cabe esperar que todos los demás sean quebrantados, pero si efectivamente es garantizado, todos ellos pueden exigirse. ${ }^{47}$

La democracia deliberativa contribuye entonces con esta concepción del estado democrático de derecho promoviendo (no excluyentemente) este ejercicio de la críticaciudadana, y señalando la necesidad de intentar vincular

45 Cfr. R. Forst, quien señala la necesidad de constituir poder discursivo en los discursos políticos para desafiar las justificaciones dadas y las estructuras de justificación existentes; la idea es analizar críticamente las relaciones políticas y sociales no justificables. Cfr. Forst, Rainer,Justificación y crítica. Perspectivas de una teoría crítica de la política (2011), Buenos Aires, Katz, 2015, pp. 125-126.

46 FG, pp. 598-599.

47 Cfr. Gargarella, Roberto, Carta abierta sobre la intolerancia. Apuntes sobre derecho y protesta, Buenos Aires, Siglo XXI Editores, 2006, p. 24. 
el discurso crítico libre, público e irrestricto orientado al consenso, con los procedimientos de fundamentación de las normas y decisiones políticas, jurídicas, etc., como así también con los correspondientes intentos de resolución de conflictos. De este modo el discurso filosófico de fundamentación de la democracia deliberativa se propone contribuir a la consolidación y a la ampliación del sistema político-democrático, así como a la autocorrección de sus falencias o desviaciones. ${ }^{48}$

\section{Reflexiones finales}

En este trabajo se intentó demostrar por qué, además de su carácter procedimental y normativo, la democracia deliberativa comporta también un carácter pragmático. El mismo se comprende a partir de la tematización de los presupuestos del principio fundamental de esta teoría política, como es el principio del discurso, conceptualmente ubicado en el marco de las implicancias teóricas del citado giro (pragmático) de la Filosofía contemporánea, aquí analizado a partir de la Filosofía del lenguaje de Habermas ( $\mathrm{y}$ en parte también de Apel). Esto expresa una concepción dialógica y comunicativa de la racionalidad, pero también de lo político, que pone énfasis en los procedimientos intersubjetivos de deliberación orientados a lograr consensos entre interlocutores activos, involucrados en la búsqueda de soluciones a los problemas que los conciernen, y que se adoptan en el marco de las instituciones democrático-formales del Estado.

La identificación de la democracia deliberativa como una teoría política que recepta (y discursivamente transforma) parte de los presupuestos del giro pragmático asumido por la Filosofía del lenguaje contemporánea, se justifica entonces a partir del señalamiento de los fundamentos teóricos del discurso argumentativo, cuyos presupuestos permiten identificar o descubrir también (parte de) las condiciones que todo régimen político tiene que reconocer para ser considerado democrático. Teniendo particularmente en cuenta este método reconstructivo de la política deliberativa, es posible a su vez inferir y

${ }^{48}$ Cfr. De Zan, Julio, "Significación moral de la democracia”, en Apel, K.-O., Cortina, Adela, De Zan, Julio y Michelini, Dorando (eds.), Ética comunicativa y democracia, Barcelona, Crítica, 1991, p. 297. 
establecer criterios generales (pero no por ello menos rigurosos) para el juicio crítico sobre el desempeño institucional de las democracias existentes, lo cual es una condición necesaria (aun cuando no sea suficiente) para mejorar la calidad democrática del estado de derecho. En efecto, si la pregunta central de la teoría política refiere a la justificación del ordenamiento político, entonces la legitimidad del mismo tiene que implicar el deber de ejercer la crítica (por parte de los organismos formales de control, pero también, y fundamentalmente, de los ciudadanos) como medio para identificary corregir el desempeño de quienes gobiernan.

La explicitación de aquellos criterios y la institucionalización de esta práctica permitirían también otorgar un mayor respaldo teórico a la justificación de un diseño institucional que acepte, por ejemplo, el control judicial de normas democráticamente sancionadas pero que contradigan principios constitucionales. Sin embargo, esta ya es una justificación que, por supuesto, requiere de un trabajo de investigación independiente.

Recibido: 10/2015; aceptado: 11/2015 